\title{
Rapid antibiotic susceptibility testing using low-cost, commercially available screen-printed electrodes
}

Stuart Hannah $^{\mathrm{a}}$, Emily Addington ${ }^{\mathrm{b}}$, David Alcorn ${ }^{\mathrm{c}}$, Wenmiao Shu ${ }^{\mathrm{a}}$, Paul A. Hoskisson ${ }^{\mathrm{b}}$ and Damion K. Corrigan ${ }^{\mathrm{a}^{*}}$

${ }^{a}$ Department of Biomedical Engineering, University of Strathclyde, 40 George Street, Glasgow, G1 1QE, United Kingdom.

${ }^{\mathrm{b}}$ Strathclyde Institute of Pharmacy and Biomedical Sciences, University of Strathclyde, 161 Cathedral Street, Glasgow, G4 0RE, United Kingdom.

${ }^{\mathrm{c}}$ Division of Anaesthesia, Royal Alexandra Hospital, Corsebar Road, Paisley, PA2 9PN, United Kingdom.

- Corresponding Author : damion.corrigan@strath.ac.uk (+44) 01415483294 (Damion Corrigan)

- Author Email Addresses : stuart.hannah@strath.ac.uk (Stuart Hannah) emily.addington@strath.ac.uk (Emily Addington) davidalcorn66@gmail.com (David Alcorn) will.shu@strath.ac.uk (Wenmiao Shu) paul.hoskisson@strath.ac.uk (Paul A. Hoskisson) damion.corrigan@strath.ac.uk (Damion Corrigan) 


\begin{abstract}
Antimicrobial resistance (AMR) is an issue of upmost global importance, with an annually increasing mortality rate and growing economic burden. Poor antimicrobial stewardship has resulted in an abundance and diverse range of antimicrobial resistance mechanisms. To tackle AMR effectively, better diagnostic tests must be developed in order to improve antibiotic stewardship and reduce the emergence of antibiotic resistant organisms. This study employs a low-cost, commercially available screen printed electrode modified with an agarose-based hydrogel deposit to monitor bacterial growth using the techniques of electrochemical impedance spectroscopy (EIS) and differential pulse voltammetry (DPV) giving rise to a new approach to measuring susceptibility. Susceptible and drug resistant Staphylococcus aureus strains were deposited onto agarose gel modified electrodes which contained clinically important antibiotics to establish growth profiles for each bacterial strain and monitor the influence of the antibiotic on bacterial growth. The results show that $S$. aureus is able to grow on electrodes modified with gel containing no antibiotic, but is inhibited when the gel modified electrode is seeded with antibiotic. Conversely, methicillin-resistant S. aureus (MRSA; the drug resistant strain) is able to grow on gel modified electrodes containing clinically relevant concentrations of antibiotic.

Results show rapid growth profiles, with possible time to results for antibiotic susceptibility $<45$ minutes, a significant improvement on the current gold standards of at least 1-2 days.
\end{abstract}

Keywords: electrochemical biosensors; antibiotic susceptibility; growth profiles; electrochemical impedance spectroscopy (EIS); antimicrobial resistance 


\section{Introduction}

The United Nations (UN) (United Nations, 2015) and the World Health Organisation (WHO) (World Health Organization, 2015) both cite AMR as an issue of worldwide concern and one of its greatest public health problems. The National Institute for Health and Care Excellence (NICE) reports AMR as an issue requiring urgent, coordinated international action (National Institute for Health and Care Excellence, 2018). It has been widely reported that by 2050, AMR will result in more than 10 million deaths per year, and if left unchecked into the future, it could account for more deaths worldwide than cancer and Type 2 diabetes (O’Neill, 2014). In recent years, approaches to tackling AMR such as the discovery and development of new antimicrobial drugs to both prevent and treat bacterial infections has stalled and the current drug pipeline is struggling to deliver (Todd et al., 2009). However, the impact of over-use and the misprescribing of antibiotics across the healthcare and agricultural sectors has resulted in the development and spread of AMR (Davies and Davies, 2010). Therefore, a One-Health approach (McEwen and Collignon, 2018) to tackling AMR is required including the development of new antimicrobial drugs, better regulation and policy, appropriate stewardship across all sectors, better infection control strategies, sanitation and animal husbandry and the development of rapid and reliable diagnostic tests to ascertain the nature of infection (bacterial or non-bacterial) to ensure effective use of antimicrobials and reduce the rate of development of resistance (Dept. of Health \& Social Care, 2019).

Current gold standards for identification of pathogens are typically based upon bacterial culture on agar plates, using classical microbiological techniques (Gilligan, 2013). Such methods are often not fast enough for modern demands, with typical processing times $\sim 1-2$ days, and are 
therefore not suitable for rapid screening at the point of care. More recent technologies, such as polymerase chain reaction (PCR) can quickly identify bacterial species, however, it requires complex sample processing and is expensive to implement (Clarridge, 2004). Therefore, new technologies that can quickly assess the effectiveness of antibiotics for a range of clinical samples, with minimal complexity of sample handling and can identify which antibiotic to prescribe are needed to help mitigate the spread of antimicrobial resistance.

Electrochemical sensors are a promising technology to investigate antibiotic susceptibility since they offer label-free detection, low-cost production, integration with other technologies including microfluidics (Nie et al., 2010) and can be integrated with simple electronics to provide real-time data and signal readout (Wang et al., 2011). One electrochemical technique suitable for low-cost integration and capable of real-time data capture is electrochemical impedance spectroscopy (EIS). With EIS, the impedance of the electrode-electrolyte interface is studied across a range of frequencies to establish information regarding the interface, its electron transfer properties and surrounding diffusional behaviour. Changes in impedance can be reflective of bacterial growth as a function of time (Ward et al., 2018; Brosel-Oliu et al., 2019). Previously, several microorganisms have been identified using EIS such as S. aureus (Ward et al., 2018), Escherichia coli (Settu et al., 2013) and Pseudomonas aeruginosa (Ward et al., 2013). Another electrochemical measurement technique of interest is differential pulse voltammetry (DPV). DPV is an example of a voltammetric technique, whereby a series of regular voltage pulses are superimposed upon a potential linear sweep or staircase waveform. It is a quick method, which eliminates the non-Faradaic charging current and can be used to sensitively investigate electron transfer to and from an electrode surface (Batchelor-McAuley et al., 2015). 
Electrochemical systems have found widespread use as biological sensors (Chauhan et al., 2017; Henihan et al., 2019; Jiang et al., 2019; Russell et al., 2019; Song et al., 2018). Additive printing processes, such as 3D-printing or screen-printing, are suitable approaches to producing costeffective electrode systems and sensors (Du et al., 2016; Tan et al., 2017). Screen printed electrodes (SPEs) feature many advantages over more traditional electrodes such as ease of fabrication and cleaning procedures, reliability, low-cost, repeatability and provide rapid time to result. SPEs are amenable to mass production at relatively low-cost compared to traditional macro or microelectrodes (Hayat and Marty, 2014). Hence, SPEs lend themselves nicely to prototyping and for the development of novel sensing technologies as reported here.

Hydrogels are increasingly finding use in devices ranging from wound dressings with controlled release of antibiotics (Tavakoli and Tang, 2017), cell stimulators for implantable bioelectronics (Han et al., 2017) to super-capacitor electrodes (Chen et al., 2013). Hydrogels are macromolecular polymer gels, made up of networks of crosslinked polymer chains. Hydrogels feature many attractive advantages for biosensor applications including their inertness to biological processes, lack of degradation, permeability to metabolites, biocompatibility, ability to withstand high temperatures and their lack of absorption by the body (Michalek et al., 2010). The porous nature of such gels including agarose and their ease of production makes them a suitable method for monitoring bacterial growth over time.

S. aureus is a Gram-positive, coccoid bacterium found in the upper respiratory tract and on the skin (Taylor and Unakal, 2019). S. aureus can cause skin infections, respiratory conditions and food poisoning (Tong et al., 2015). S. aureus is a useful bacterium to study the emergence of 
antibiotic-resistant strains with MRSA being a common problem in the clinic (Harkins et al., 2017). A useful biosensor should be able to distinguish between susceptible and resistant strains of $S$. aureus with the aim of providing the correct treatment to the patient as rapidly as possible. Since MRSA is a leading cause of surgical, hospital and community acquired infection (Coll et al., 2017) distinguishing between antibiotic-sensitive $S$. aureus vs MRSA has been employed as a proof of concept towards development of a rapid, point of care test to improve antibiotic stewardship.

In this paper, to assess antibiotic susceptibility, electrochemically derived growth profiles for antibiotic-sensitive $S$. aureus and MRSA are explored using electrodes modified with agarosegel deposits containing growth medium and different concentrations of antibiotics. The optimum electrochemical parameters were evaluated with regards to sensitivity of antibiotic-sensitive $S$. aureus and MRSA growth, and reported as a function of bacterial growth for both strains tested and in the presence and absence of two commonly used antibiotics, amoxicillin and oxacillin.

\section{Material and methods}

\subsection{Methodology}

Commercially available Gold (Au) screen-printed electrodes (SPEs) with on-chip silver reference and gold counter electrodes were obtained from DropSens (Oviedo, Spain) (ref 223BT).

Gels for electrode characterisation contained agarose, Miller LB Broth, $200 \mathrm{mM}$ KCL + $1 \mathrm{mM}$ $\mathrm{Fe}[\mathrm{CN}]_{6}{ }^{3-}+1 \mathrm{mM} \mathrm{Fe}[\mathrm{CN}]_{6}{ }^{4-}$ (Ferri-Ferro Cyanide (FF-C) solution) with some containing antibiotics (amoxicillin or oxacillin at $8 \mu \mathrm{g} / \mathrm{ml}$ in DI water). All chemicals were purchased from 
Sigma Aldrich, Dorset, UK. Gel components were initially mixed at room temperature, and then autoclaved at $121^{\circ} \mathrm{C}$ to both sterilise and allow the agarose to mix. Upon cooling, the gels hardened, and were re-melted prior to deposition on the electrode. Antibiotics were added to the gels upon cooling to avoid inactivation at high temperature.

Bacterial plates for culture contained LB media + agar (Sigma Aldrich). Bacterial strains Staphylococcus aureus (ATCC 29213) and methicillin-resistant Staphylococcus aureus (MRSA: ATCC 43300) were streaked out onto LB agar-plates from a freshly prepared frozen glycerol stock of each (stored at $-80^{\circ} \mathrm{C}$ ). Single colonies were used to inoculate overnight cultures of Lysogeny Broth $\left(5 \mathrm{ml}\right.$ at $\left.37^{\circ} \mathrm{C}\right)$. Bacteria from the overnight cultures were directly pipetted onto the gels on the electrode surface, at a volume of $5 \mu 1$ and a bacterial concentration $\sim 10^{7} \mathrm{CFU} / \mathrm{ml}$ (CFU - colony forming unit) giving a final count on the sensor of $\sim 50,000$ CFUs. LB agarose was prepared as LB but replacing agar with agarose.

Prior to measurement, all electrodes were cleaned using an electrochemical method involving immersion of the electrode in DI water $+200 \mathrm{mM} \mathrm{KCL}+1 \mathrm{mM} \mathrm{Fe}[\mathrm{CN}]_{6}^{3-}+1 \mathrm{mM} \mathrm{Fe}[\mathrm{CN}]_{6}^{4-}$ (Ferri-Ferro Cyanide (FF-C) solution) and performing cyclic voltammetry (CV) over a potential range of $-0.3 \mathrm{~V}$ to $+0.8 \mathrm{~V}$ for 10 scans at $100 \mathrm{mV} / \mathrm{s}$. After cleaning, the electrodes were rinsed with DI water and dried using an aerosol gun prior to use.

\subsection{Characterisation}

Electrode measurements were performed using a three-electrode cell. DropSens SPEs are designed with the counter, reference and working electrodes together on a single chip, which 
eliminates the need to incorporate external counter and reference electrodes. All measurements were carried out using a potentiostat (PalmSens PS4, PalmSens, Houten, Netherlands).

Scanning electron microscope (SEM) (TM-1000, Hitachi, Tokyo, Japan) images were taken of the Au electrode surface with and without the gel to gain an impression of the surface profile. SEM images were performed by scanning a $30 \times 30 \mu \mathrm{m}$ area, at a magnification of $\mathrm{x} 5.0 \mathrm{k}$.

CV measurements were nominally performed by sweeping the potential between $-0.3 \mathrm{~V}$ to $0.5 \mathrm{~V}$ with reference to the Ag electrode three times. Analysis was performed using the third measurement. Electrodes were characterised using differential pulse voltammetry (DPV) across the same potential range as CV and the peak current $\left(I_{p k}\right)$ was extracted. Electrodes' EIS response was measured across a frequency range between $100 \mathrm{kHz}$ and $0.1 \mathrm{~Hz}$ at open circuit using an amplitude of $10 \mathrm{mV}$ rms. The generated Nyquist and Bode impedance plots were fitted to the Randles' equivalent circuit to extract various impedance parameters. Figure 1 (b) shows an example EIS response of 1mM FF-C on the Au electrode, with the Randles' equivalent circuit used for data fitting shown in the inset.

Prior to bacterial deposition on the electrode surface, CV, DPV and EIS measurements were performed on the modified electrodes. Once deposited, the gel was left to partially stabilise at $37^{\circ} \mathrm{C}$ for 10 minutes. Then, $5 \mu 1$ of either S. aureus or MRSA culture from an overnight culture $\left(\sim 10^{7} \mathrm{CFU} / \mathrm{ml}\right)$ was pipetted on top of the gel. DPV and EIS measurements were performed every 5 minutes using a measurement script, and extracted parameters were plotted as a function of time up to a maximum of 2.5 hours of bacterial growth post deposition. All bacterial growth 
experiments were performed at $37^{\circ} \mathrm{C}$ within a humidity chamber contained within an incubator

(Genlab Ltd, Widnes, UK). For every growth curve produced, a minimum of three experiments were performed which were found to be entirely internally self-consistent, this low intra experimental variation gave confidence that the reported growth effects and relative differences were reproducible. Representative data is displayed and error bars are not shown because inter experimental variation was higher thus making error bars unrepresentative of true effects. Inter experimental variation was high because of sensor to sensor variation and variations in the cleaning and gel deposition processes, all of which will be the subject of future standardisation experiments. Figure 1 (a (i)) displays the measurement setup adopted, including the electrode modified with a gel deposit connected to the potentiostat and related software for electrochemical measurements.

Information regarding determination of minimum inhibitory concentrations (MIC) of antibiotics is included in the supporting information for reference.

\section{Results and discussion}

\subsection{Electrodes and electrochemical measurements}

A commercially available electrode was chosen for this study in order to benefit from its low$\operatorname{cost}(<£ 2$ each), and ease of integration with the developed measurement setup. The electrode chosen was a $1.6 \mathrm{~mm}$-diameter screen printed electrode (SPE) (DropSens, Oviedo, Spain). The SPE (33 mm x $10 \mathrm{~mm}$ x $0.5 \mathrm{~mm}$ (length $\times$ width $\times$ height)) consists of three main parts: a 1.6 mm-diameter Au working electrode, Au counter electrode and a silver (Ag) reference electrode. The electrical contacts on the electrode are made of Ag. Previously, these Au SPEs have been 
used to produce disposable nucleic acid biosensors (Kuralay et al., 2015) and for the detection of volatile fatty acids (Ndiaye et al., 2016) amongst other things.

Figures 1 (a) (iii) and (iv) show SEM images of the bare Au electrode surface (iii) and the electrode surface containing a $1 \%$ agarose gel deposit (iv). SEM images of the electrodes show a highly irregular surface profile, which contains deep voids on the micron scale across the surface and non-homogenous particle sizes. It is clear from the SEM images that the gel deposit has no effect on the electrode surface itself, only a subtle blurring of the image is visible when the gel is present which is to be expected.

Figure 1 (b) shows a typical EIS measurement performed from $100 \mathrm{kHz}$ to $0.1 \mathrm{~Hz}$ using ferricyanide, with the inset displaying the classical Randles' equivalent circuit model used to fit the EIS data. By fitting the data to this circuit, a number of analytical parameters can be extracted, including the solution resistance $\left(R_{S O L N}\right)$, charge transfer resistance $\left(R_{C T}\right)$ and double layer capacitance $\left(C_{D L}\right)$. In addition, through extraction of the EIS Bode plot, information regarding the impedance $(Z)$ at different frequencies and the phase angle can be extracted. A wide range of parameters were found to change over time as a function of microbial growth, and subsequent results present data extracted using the most sensitive and convenient parameters. In addition, the DPV peak current $\left(I_{p k}\right)$ can also be investigated.

\subsection{Gel production and electrode preparation}

Agarose was chosen as a suitable gel material, due to its UV transparency and low toxicity (Science Direct, 2019). Agarose is one of the two main components of agar, and is commonly used in electrophoresis, to separate large molecules such as DNA (Thermo Fisher Scientific, 
2019). Agarose performs best in gel form at relatively low concentrations in solution, for example, $1 \%$ gels are commonly used to provide good separation and resolution of large DNA fragments during electrophoresis (Sabath et al., 1976). It was found that agarose concentrations below $1 \%$ failed to produce completely spherical gels, a key requirement for application across the entire sensor working electrode surface. Even small changes in gel concentration can affect its properties such as gelation and other properties of the $3 \mathrm{D}$ construct can be significantly affected, therefore, three similar concentrations of agarose were tested $(1 \%, 1.5 \%$ and $3 \%)$ to evaluate gel stability and ease of deposition, with the aim to select the optimum agarose concentration. Figure 1 (a (ii)) shows an example gel deposit on the electrode. Figure 1 (c) shows the electrochemical response (i) CV and (ii) EIS of the three agarose gels tested. Each gel contained FF-C to ensure a Faradaic signal could be measured, i.e. the FF-C redox mediator could permeate the gel effectively to reach the working electrode surface. A motivation for adding a redox agent to the gel was to expand the number of electrochemical parameters which could be investigated during microbial growth. For comparison, Figure S1 of the supporting information displays the CV and EIS data relating to the bare electrode surface in comparison with the $1 \%$ gel. Referring to the CV response (Figure 1 (c) (i)), as the concentration of agarose in the gel is increased, the $\mathrm{CV}$ response displayed reduced current, with greater separation between the oxidation and reduction peaks, indicating an improved electrochemical response for the $1 \%$ gel. This is confirmed with EIS measurements (Figure 1 (c) (ii), whereby $R_{C T}$ increases significantly as agarose concentration is increased. The Randles-Sevcik equation (Eqn. 1) can be used to describe the effect of scan rate on peak current for cyclic voltammetry measurements.

$i_{p}=0.4463 n F A C\left(\frac{n F v D}{R T}\right)^{1 / 2}$ 
Where $i_{p}$ is the maximum current in amps, $n$ is the number of electrons transferred in the redox event, $A$ is the electrode area in $\mathrm{cm}^{2}, F$ is the Faraday Constant in $\mathrm{C} \mathrm{mol}^{-1}, D$ is the diffusion coefficient in $\mathrm{cm}^{2} \mathrm{~s}^{-1}, C$ is the concentration in $\mathrm{mol} \mathrm{cm}^{-3}, v$ is the scan rate in $\mathrm{V} \cdot \mathrm{s}^{-1}, R$ is the gas constant in $\mathrm{J} \mathrm{K}^{-1} \mathrm{~mol}^{-1}$ and $T$ is the temperature in $\mathrm{K}$. Various parameters can be extracted from $\mathrm{CV}$ curves including $i_{p}$ and peak potential separation $\left(\Delta E_{p}\right)$ (Table 1). It is clear that as the concentration of agarose in the gel is increased, there is a significant increase in the peak separation, indicative of reduced reversibility at higher agarose concentrations.

From the EIS data, Equation 2 can be used to find the diffusion coefficient of the ionic species (FF-C) or the area of the electrode:

$$
R_{C T}=\frac{4 R T l}{A D F^{2} C}
$$

Where $l$ is the diffusion length and $R_{C T}$ is the charge transfer resistance in ohms extracted from a fit of the measured EIS data. By rearranging Eqn. 2 for $D$, the diffusion coefficient at each agarose concentration can be determined, and used to assess the influence agarose has on the effect of FF-C to penetrate to the electrode surface under mass transport conditions. ' $D$ ' was chosen as the parameter of interest in this case and the electrode area was kept constant due to the cross-linked nature of the gel meaning ' $D$ ' would likely have a greater impact on the diffusional behaviour rather than the resulting electrode area, however, it is acknowledged that equally ' $D$ ' could have been fixed and the area as a function of agarose concentration could have 
been investigated instead. Table 1 displays the value of $D$ extracted at each agarose concentration, ranging from $4.15 \times 10^{-9}$ for $1 \%$ agarose to $7.64 \times 10^{-10} \mathrm{~cm}^{2} \mathrm{~s}^{-1}$ for $3 \%$ agarose. The $1.5 \%$ agarose displays a significantly higher value of $2.23 \times 10^{-6} \mathrm{~cm}^{2} \mathrm{~s}^{-1,}$ however, it also displays a greater $R_{S O L N}$ from the EIS measurement which helps explains the difference seen in the calculated value of $D$. From Table 1 it can also be noted that the $\chi^{2}$ value, or the 'goodness of fit', statistical test is around an order of magnitude higher for the $1.5 \%$ agarose, compared to the other two agarose concentrations tested. This is an indication that while the $1.5 \%$ gel measurement may not be entirely consistent with the others, it is still useful as means to show the gel development. Due to the improvement in electrochemical response seen at the lower agarose concentration and it's ease at forming a reproducible spherical gel on the electrode surface, the 1 $\%$ gel was chosen for all subsequent experiments.

When working with hydrogels, the deposition method is an important factor to consider because this affects reproducibility and uniformity of gel deposits from run-to-run. Dip-coating is a useful method to produce a thin gel layer on the electrode surface, however, it requires a large volume of material, making it expensive. When dip-coating manually, it is also difficult to achieve a precise layer-thickness each time, since the resulting layer depends upon the speed with which the electrode is removed from the gel solution. Conversely, drop-casting is a simpler method and only uses the exact volume of material required, reducing the cost. A simple method of dropcasting is to pipette gels using a specific volume, which results in more reproducible deposits than dip-coating. The CV and EIS response of three gels deposited using dip-coating and dropcasting (10 and $20 \mu \mathrm{l})$ are shown in Figure 1 (d) (i) and (ii) respectively. Two drop-casting volumes were tested; pipetting $10 \mu 1$ onto the WE only, and then $20 \mu 1$ pipetted onto all three 
electrodes (WE/RE/CE). From the CV response and the EIS, the $20 \mu 1$-gel deposit appears to produce the most consistent electrochemical response. In terms of the CV curves, the $20 \mu 1$-gel deposit produces around a 10 times greater peak current, and the peak separation is closer to the theoretical limit $(\sim 59 \mathrm{mV})$ at $85.55 \mathrm{mV}$, compared to $140.94 \mathrm{mV}$ for the $10 \mu \mathrm{l}$-gel deposit. It also provides an acceptably low $R_{C T}$ enabling satisfactory electron transfer between the gel deposit and the electrode surface. Therefore, for subsequent measurements, at least $20 \mu \mathrm{l}$ of gel was pipetted onto the SPE, to ensure coverage of all three electrodes on the chip. The larger gel volume also enables longer measurement times, since smaller volumes have a tendency to evaporate more readily during microbial growth.

Table 1 provides a summary of the electrochemical measurement data extracted from the gel development stage. The majority of the parameters are taken from EIS data, fitted to the Randles' equivalent circuit, although additional data from CV and DPV measurements is also included for comparison. Table 1 contains the data extracted from electrochemical techniques performed during the gel development stage, in particular, looking at the effect of agarose concentration and gel deposition method on the obtained gel deposit. The data confirms that 20 $\mu 1,1 \%$-agarose gels drop-casted onto the SPE provided the optimum measurement conditions. 
a (i)
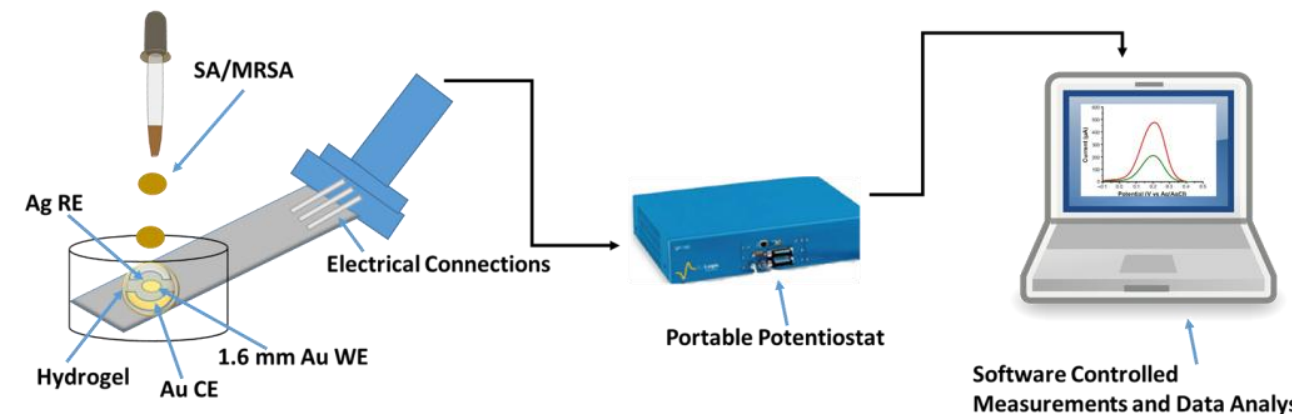

Measurements and Data Analysis

a (ii)

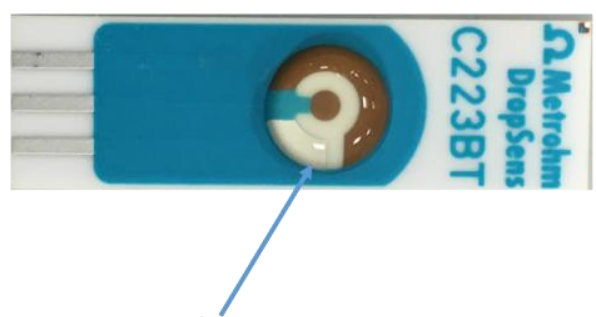

a (iii)

Gel Deposit

b

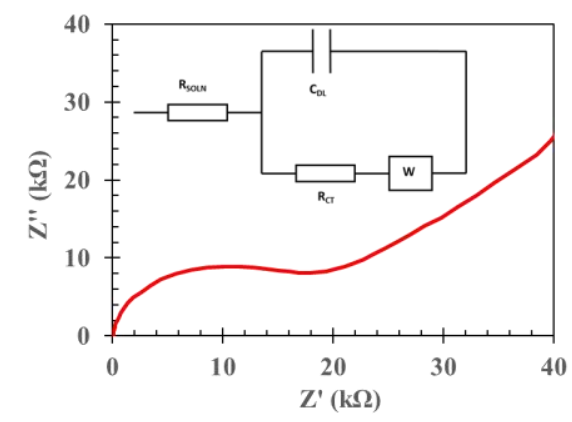

a (iv)
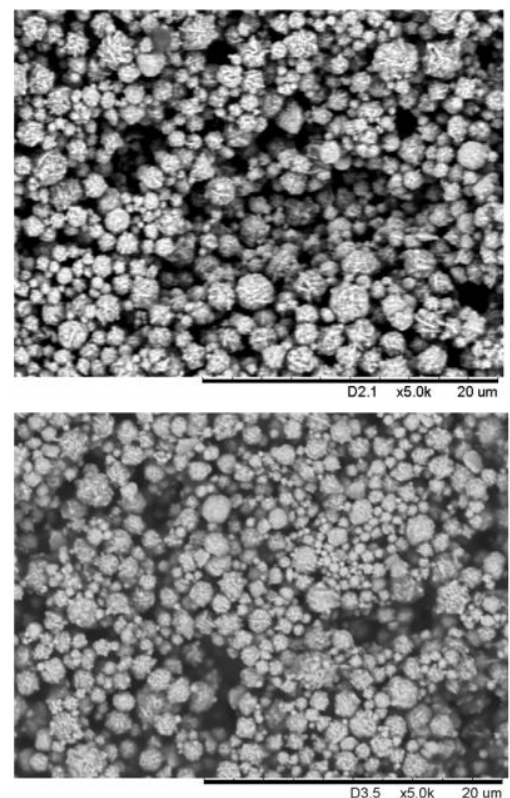

c (i)

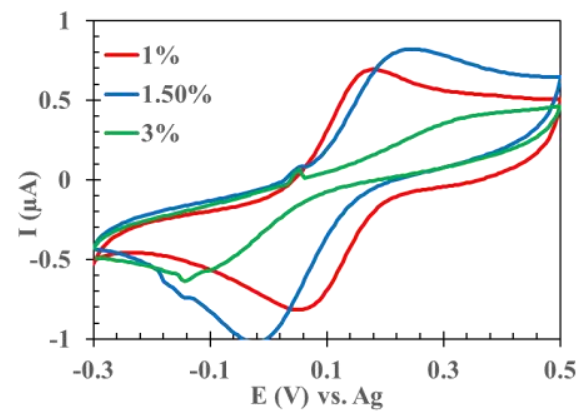

c (ii)

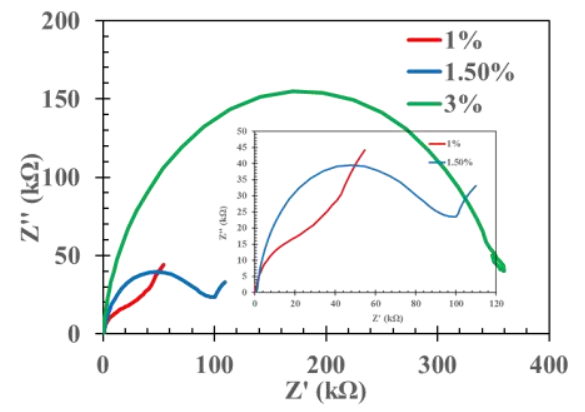

d (i)

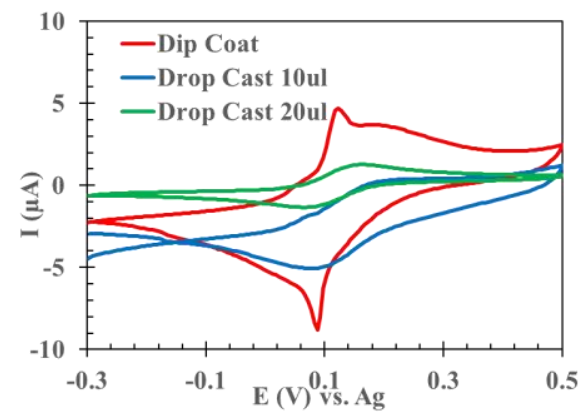

d (ii)

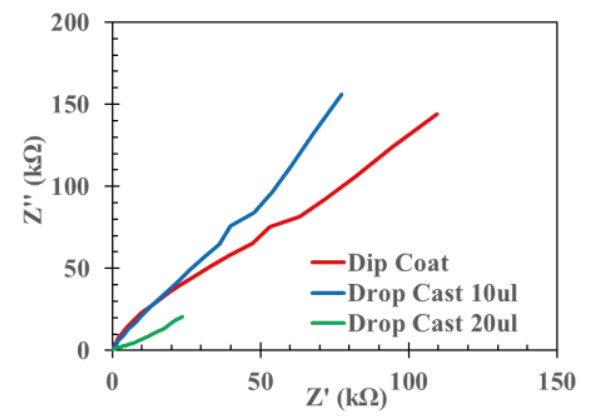


Figure 1. (a (i)) Experimental setup featuring Au DropSens electrode modified with gel deposit containing agarose, LB and FF-C and some with antibiotic. Schematic depicts bacteria being pipetted onto electrode, and electrochemical measurements being performed using a potentiostat. (a) (ii) Electrode surface modified with agarose gel deposit. (a) (iii) SEM image of bare Au DropSens electrode. (a) (iv) SEM image of Au DropSens electrode with $1 \%$ agarose gel deposit. (b) Typical electrochemical impedance spectroscopy (EIS) measurement of $1 \mathrm{mM} F \mathrm{~F}-\mathrm{C}$ on Au SPE. Inset shows Randles' equivalent circuit model used to fit EIS data. (c) CV (i) and EIS (ii) measurements on different concentrations of agarose gel containing $\mathrm{F}-\mathrm{F}+\mathrm{KCl}$. Inset in (c) (ii) shows 1 and $1.5 \%$ gels only for clarity. (d) CV (i) and EIS (ii) measurements comparing gel deposition techniques.

Table 1. Summary of data shown in Figure 1. Table shows CV, EIS and DPV measurement data extracted from gel development experiments. Measurement parameters include $R_{S O L N}, R_{C T}, C_{D L}, Z(100 \mathrm{kHz})$, Phase, DPV $I_{p k}, C V i_{p k}$, $C V \triangle E_{p}$ and $D$.

\begin{tabular}{|c|c|c|c|c|c|c|c|c|c|c|}
\hline $\begin{array}{c}\text { Experiment: } \\
\text { Agarose } \\
\text { Concentration } \\
(\%)\end{array}$ & $\frac{\underline{R}_{S O L N}}{(\Omega)}$ & $\begin{array}{l}\underline{R_{C T}} \\
\underline{(\mathrm{k} \Omega)}\end{array}$ & $\frac{C_{D L}}{(\mu \mathrm{F})}$ & $\chi^{2}$ & $\frac{\underline{Z \text { at }}}{\frac{100}{\mathrm{kHz}}}$ & $\frac{\text { Phase }}{\left({ }^{\circ}\right)}$ & $\frac{\operatorname{DPV} I_{p k}}{(\mu \mathrm{A})}$ & $\frac{C V i_{p k}}{(\mu \mathrm{A})}$ & $\frac{\mathrm{CV} \Delta E_{p}}{(\mathrm{mV})}$ & $\begin{array}{c}\left.\frac{D}{\left(\mathrm{~cm}^{2}\right.} \mathrm{s}^{-1}\right) \\
\end{array}$ \\
\hline $1 \%$ & 299.80 & 24.25 & 1.84 & 0.0007 & $\begin{array}{c}415.4 \\
0\end{array}$ & 22.42 & 0.46 & 0.69 & 110.40 & $4.15 \times 10^{-9}$ \\
\hline $1.5 \%$ & 731.40 & 94.03 & 0.57 & 0.0101 & $\begin{array}{c}658.5 \\
2\end{array}$ & 6.58 & 0.19 & 0.82 & 281.95 & $2.23 \times 10^{-6}$ \\
\hline $3.0 \%$ & 249.60 & 338.8 & 0.24 & 0.0011 & $\begin{array}{c}246.6 \\
5\end{array}$ & 6.18 & 0.02 & 0.41 & 498.52 & $7.64 \times 10^{-10}$ \\
\hline $\begin{array}{l}\text { Experiment: } \\
\text { Gel Deposition } \\
\text { Method }\end{array}$ & $\frac{\underline{R}_{S O L N}}{\underline{(\Omega)}}$ & $\begin{array}{l}\underline{R}_{C T} \\
\underline{(\mathrm{k} \Omega)}\end{array}$ & $\frac{C_{D L}}{(\mu \mathrm{F})}$ & $\chi^{2}$ & $\frac{\underline{Z \text { at }}}{\frac{100}{\mathrm{kHz}}}$ & $\frac{\text { Phase }}{\left({ }^{\circ}\right)}$ & $\frac{\operatorname{DPV} I_{p k}}{(\mu \mathrm{A})}$ & $\frac{C V i_{p k}}{(\mu \mathrm{A})}$ & $\frac{\mathrm{CV} \Delta E_{p}}{(\mathrm{mV})}$ & $\begin{array}{c}\left.\frac{D}{\left(\mathrm{~cm}^{2}\right.} \mathrm{s}^{-1}\right) \\
\end{array}$ \\
\hline Dip Coat & 226.50 & I & 2.04 & 0.0024 & $\begin{array}{c}217.4 \\
2\end{array}$ & 1.66 & 5.26 & I & I & I \\
\hline $\begin{array}{l}\text { Drop Cast } \\
(10 \mu 1)\end{array}$ & 191.40 & 0.08 & 3.38 & 0.0008 & $\begin{array}{c}185.6 \\
4\end{array}$ & 1.87 & 2.91 & 0.102 & 140.94 & I \\
\hline $\begin{array}{l}\text { Drop Cast } \\
(20 \mu 1)\end{array}$ & 242.2 & 3.91 & 1.36 & 0.0009 & $\begin{array}{c}242.4 \\
1\end{array}$ & 4.37 & 1.42 & 1.26 & 85.55 & I \\
\hline
\end{tabular}


Additional data containing electrochemical data concerning the effect of different antibiotics as well as gel components LB Broth and amoxicillin can be found in the supporting information (Figure S2 and Table S1).

\subsection{Bacterial growth profiles}

Following gel development, the next step was to monitor bacterial behaviour once loaded onto the electrode. Initially, a baseline measurement was recorded, i.e. without any bacteria present, to characterise the electrochemical signal from the gel modified sensor as a function of time (up to 150 minutes). EIS/DPV measurements were taken every 5 minutes following gel deposition, and three curves were produced; a baseline, bacterial growth without antibiotic present, and bacterial growth in the presence of an antibiotic (amox or oxa). Antibiotics amox and oxa were prepared at a concentration of $8 \mu \mathrm{g} / \mathrm{ml}$ after evaluating their MICs to ensure MRSA could in fact grow on the electrode, but crucially that $S$. aureus growth would be inhibited. Differences between antibiotic-sensitive $S$. aureus and MRSA growth on antibiotic-free and antibiotic-containing gels were sought in order to assess susceptibility. From all the various parameters extracted from EIS and DPV measurements, it was found that the impedance $(Z)$ at $100 \mathrm{kHz}$ provided the most sensitive response to bacteria, and therefore is displayed in this paper.

\subsection{Amoxicillin modified sensor behaviour}

Figure 2 (a) (i) shows growth curves of S. aureus on the gel-modified sensors for 150 minutes, until evaporation. It is clear that $S$. aureus pipetted onto the amoxicillin-infused sensor produces an almost identical response to the baseline response, i.e. the situation where bacteria were not present in the $5 \mathrm{ul}$ aliquot mimics inoculation with $S$. aureus, indicating inhibited growth. This data correlates with what was found for $S$. aureus growth on agarose-plates infused with 
amoxicillin and incubated and assessed using traditional microbiology techniques (Figures S5 and S6, Supporting Info) and gives confidence that the electrochemical technique correlates with the benchmark microbiology work. Conversely, S. aureus loaded onto the gel without amoxicillin gave a different $Z$ profile, indicating bacterial growth as a function of time. The baseline data was then subtracted from the two curves to properly visualise the change in $Z$ as a function of time, to pinpoint the bacterial growth period. Figure 2 (a) (ii) shows the result of the baseline subtraction for the sensor infused with amoxicillin and the sensor without for $S$. aureus growth. Interestingly, the $Z$ for $S$. aureus on the amoxicillin-laden gel is unchanging between 30 and 100 minutes, whereas, for $S$. aureus on the gel containing no antibiotic, $Z$ increases by around $200 \%$, indicating changes in the composition of the gel, most likely arising from bacterial metabolism of its constituents, explaining why changes in $Z$ are apparent on time frames shorter than the organism's doubling time. The time period of $30-100$ minutes was chosen for detailed analysis since before and after this timeframe, the gel was seen to undergo initial structural changes and latterly evaporation changes, influencing the concentration of FF-C in the gel and ultimately, affecting the electrochemical behaviour.

Next, a resistant $S$. aureus strain (MRSA) was investigated in the same manner as for the sensitive S. aureus strain. Figure 2 (b) (i) shows growth profiles for MRSA on a sensor modified with amoxicillin at a concentration close to the MIC value $(8 \mu \mathrm{g} / \mathrm{ml})$ and a gel without amoxicillin present. This time, MRSA behaviour (on the amoxicillin-laden gel) does not imitate the baseline like $S$. aureus was found to, but instead displays a more similar (increasing) $Z$ gradient akin to when MRSA growth was measured on the non-antibiotic gel. This is confirmed when the baseline curve is subtracted from the data (Figure 2 (b) (ii)). In this instance, the 
MRSA baseline subtracted data for each gel shows a steady increase over time, indicating there is hindered growth occurring in the presence of amoxicillin. Over 30-100 minutes, the change in $Z$ for the gel without amoxicillin is $\sim 24 \Omega$, whereas the change for the $8 \mu \mathrm{g} / \mathrm{ml}$ amoxicillin-gel is $\sim 11 \Omega$. This is to be expected since antibiotic resistance is not always a "binary situation" (Sabath et al., 1976) and the antibiotic does still have an effect on the resistant strain by retarding growth. Clinically this manifests as the ability to survive in the presence of the intended therapeutic dose of antibiotic. However, what is clear, is that a difference between the $S$. aureus and MRSA strains could be seen for the same amoxicillin concentration, allowing these two strains to be distinguished within 100 minutes, if not less. To verify the effect of amoxicillin on the MRSA strain, a growth profile for MRSA on amox infused gel at a concentration significantly greater than the MIC $(50 \mu \mathrm{g} / \mathrm{ml})$ was produced. At this concentration, amoxicillin should completely inhibit MRSA growth, and the growth profile confirms this is indeed the case since it displays a very similar profile to the baseline measurement (Figure 2 (b) (i)). Once this higher antibiotic concentration measurement is subtracted from the baseline (Figure 2 (b) (ii)), it is evident that the concentration of amoxicillin has a significant effect on the growth of MRSA. Unlike the amoxicillin at $8 \mu \mathrm{g} / \mathrm{ml}$ which displays hindered growth, there is virtually no change in $Z$ for MRSA on $50 \mu \mathrm{g} / \mathrm{ml}$ amoxicillin between the same $30-100$ minutes' time period. The subtracted growth profile is completely flat, indicative of inhibited MRSA growth at this antibiotic concentration. Additionally, growth profiles of MRSA on sensors modified with amoxicillin gels at each antibiotic concentration $(8 \mu \mathrm{g} / \mathrm{ml}$ and $50 \mu \mathrm{g} / \mathrm{ml})$ and without antibiotic were produced using DPV for comparison to $Z$ growth profiles (Figure S7 Supporting Info). In this case, the DPV peak current $\left(I_{p k}\right)$ was plotted as a function of time, and it is clear that $I_{p k}$ displays a similar growth profile to $Z$. The sensor modified with a gel containing no amoxicillin 
displays a similar profile (steeper gradient) to the sensor modified with an amoxicillin-laden gel at $8 \mu \mathrm{g} / \mathrm{ml}$, whereas for the case of amoxicillin at $50 \mu \mathrm{g} / \mathrm{ml}$, once this measurement has been subtracted from the baseline, it is almost completely flat, once again indicative of inhibited MRSA growth at this antibiotic concentration. Despite the ease of which DPV measurements can be taken, it appears to be more sensitive to changes in gel composition effects over time (E.g. drying), and therefore $Z$ is a more sensitive and useful parameter for bacterial growth profiling.

a (i)

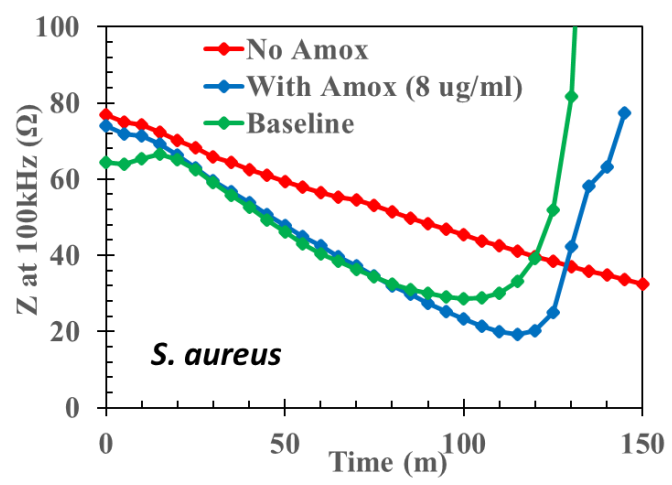

b (i)

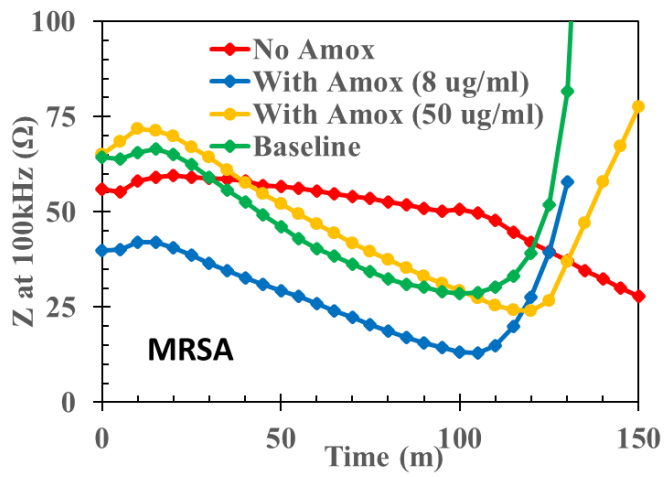

a (ii)

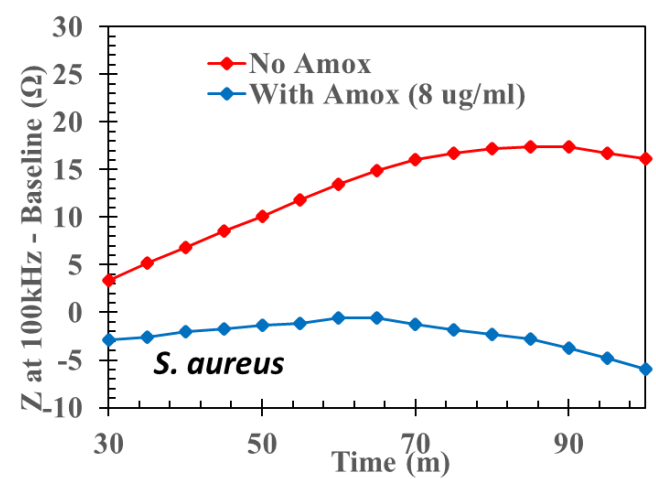

b (ii)

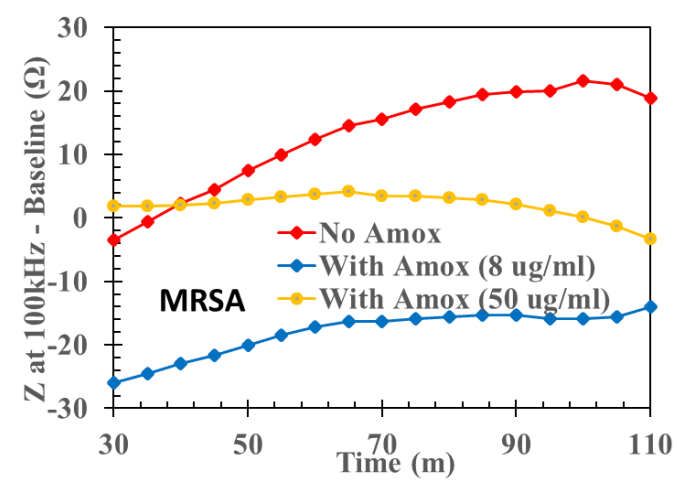

Figure 2. Bacterial growth curves of sensitive $S$. aureus (a) and MRSA (b) strains on gels infused with and without amoxicillin ( $8 \mu \mathrm{g} / \mathrm{ml}$ (and $50 \mu \mathrm{g} / \mathrm{ml}$ for MRSA)) and baseline (no bacteria). (i) $\mathrm{Z}$ at $100 \mathrm{kHz}$ and (ii) $\mathrm{Z}$ at $100 \mathrm{kHz}-$ baseline curve. 


\subsection{Oxacillin modified sensor behaviour}

The amoxicillin results were then compared with another antibiotic, oxacillin, which is now more commonly used to test for resistance than methicillin (Centers for Disease Control and Prevention, 2019), therefore oxacillin was used to perform benchmark MIC concentration assays (Figure S4, Supporting Info) as well as electrode growth measurements to investigate S. aureus and MRSA growth. Figure 3 (a) (i) presents growth curves of $S$. aureus on an oxacillin-laden gel and a gel containing no antibiotic, as well as the baseline (gel only) curve for comparison. In a similar manner to the results obtained using amoxicillin, S. aureus grown on the oxacillin-laden gel produced a very similar $Z$ profile to the baseline measurement where no bacteria were deposited in the $5 \mu 1$ aliquot, an indication that oxacillin at $8 \mu \mathrm{g} / \mathrm{ml}$ is capable of inhibiting $S$. aureus growth. On the other hand, S. aureus on the gel with no oxacillin displays a significantly different $Z$ profile, representative of $S$. aureus growth. Figure 3 (a) (ii) displays the growth data with the baseline signal subtracted. In this case, there is a clear distinction between $S$. aureus grown on oxacillin and without. In the case of the no antibiotic gel, $Z$ increases by $\sim 20 \Omega$, whereas for the gel with oxacillin, $\mathrm{Z}$ remains fairly flat by comparison up to $\sim 70$ minutes, then a drop-off in $\mathrm{Z}$ begins, likely an effect of the gel evaporation process, since for up to 70 minutes in the no antibiotic gel case, there is clear growth in $Z$ from as early as 30 minutes after initial bacteria deposition.

MRSA was also tested on gels infused with and without oxacillin. Figure 3 (b) (i) shows the growth curves produced alongside the baseline data. Unlike S. aureus, MRSA growth curves look very similar for gels with and without oxacillin, as previously shown with amoxicillin. There are again subtle differences for the MRSA samples grown on gels containing different 
levels of oxacillin and these manifest in the gradient of the electrochemical bacterial growth profile. This is to be expected, as explained earlier, drug resistance is a complex phenomenon and in the case of MRSA, the organism is still susceptible to high concentrations of beta-lactam antibiotics. Clinically the resistance manifests as an ability to grow above typical MIC values since these are the conditions under which resistance develops. The sensor corroborates this by showing the electrochemical growth profile is most striking in the absence of any antibiotic but is also capable of showing hindered growth in the case of these MRSA experiments using sensors modified with antibiotic concentrations close to the MIC. These findings show that in principle the gel/antibiotic modified electrode sensor has the potential to evaluate bacterial growth and identify drug/dose relationships which retard growth. There is clear potential for antibiotic growth profiling and susceptibility testing using this approach.

a (i)

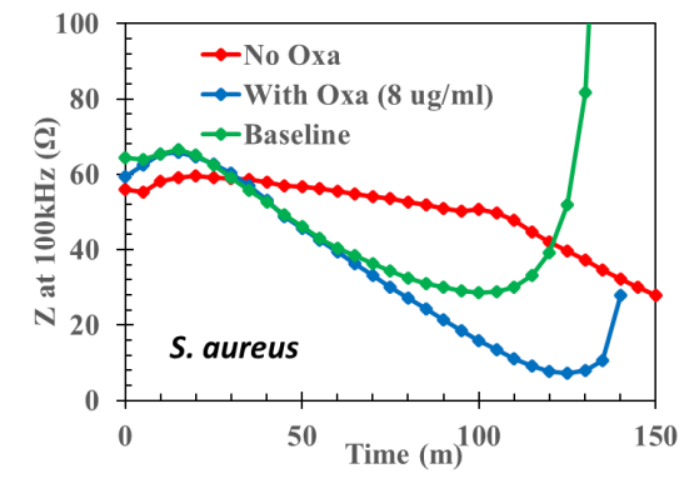

b (i)

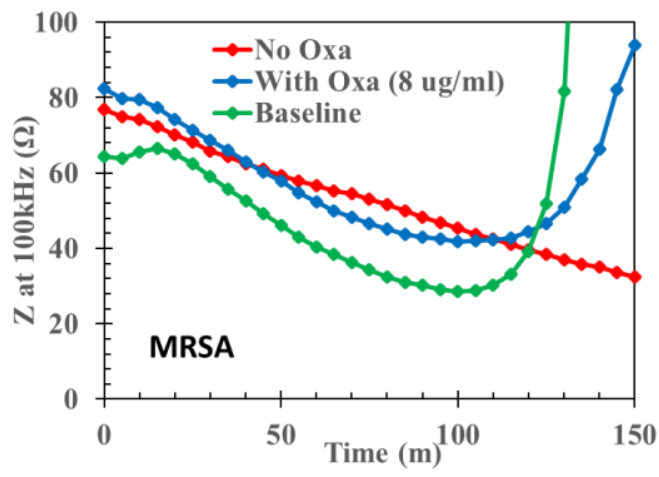

a (ii)
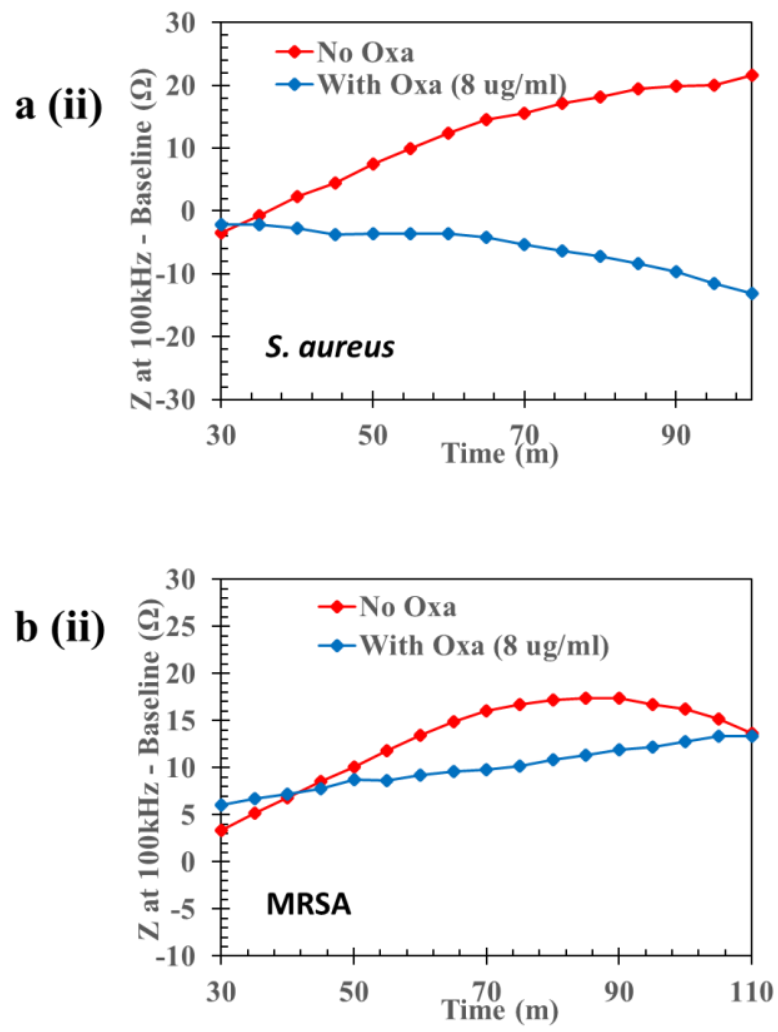
Figure 3. Bacteria growth curves of sensitive S. aureus (a) and MRSA (b) strains on gels infused with and without oxacillin (Oxa) (8 $\mu \mathrm{g} / \mathrm{ml}$ ) and baseline (no bacteria). (i) $\mathrm{Z}$ at $100 \mathrm{kHz}$ and (ii) $\mathrm{Z}$ at $100 \mathrm{kHz}$ - baseline curve.

A clear advantage of this approach to electrochemical measurement of antibiotic susceptibility is the low cost nature of the sensor developed and the relative simplicity of the approach taken. Classical microbiological techniques such as agar plates, growth profiling, MIC assays and susceptibility testing are still in routine use because they work well in the clinical environment. Uptake of PCR based approaches has been slower and this is for a variety of reasons which include: difficulties in persuading health agencies to innovate, PCR reagents can be expensive and also genetic indicators of resistance may be present and identifiable but that does not always convert into the bacteria being resistant (other genetic factors play a role in determining resistance). The biosensor community has often concentrated on genetic profiling when developing sensors for AMR, here the pragmatic approach of phenotypic testing has been shown to have some benefits. First of all, electrochemical measurements can be recorded on gelmodified electrodes without the need to submerge the sensor into a liquid, secondly there is no need to chemically functionalise the sensor surface with a DNA recognition element (this can be a tremendous source of measurement variation for DNA biosensors) and thirdly a wide range of electrochemical parameters can be measured and used to interpret the bacterial growth profile. The ability to easily modify the gels (antibiotic concentration, nutrient composition, redox agents etc.) in combination with the ability to array a number of electrodes on a single chip means this approach has significant potential for use in clinical antibiotic susceptibility testing. 
Finally, the performance of this sensor can be compared with previously published studies such as Ward et al., 2018 and Brosel-Oliu et al., 2019 where S. aureus and E. coli were being detected respectively. In both of these cases, $\sim 500,000$ CFUs was the final cell count on the sensors, which is similar to our inoculation of $\sim 50,000$ CFUs. Our sensor provides good sensitivity at this cell density, and displays a time to result which is comparable to these previously published studies. Additionally, further modifications are being made which will enhance the systems sensitivity.

\section{Conclusions}

A low-cost, commercially available, gold screen printed electrode has been used to monitor bacterial growth following modification and using the electrochemical techniques of EIS and DPV. A hydrogel deposit which covered all three electrodes was developed using a common polysaccharide agarose, to benefit from its porous nature, biocompatibility and ability to drop cast. Agarose gels were developed containing different antibiotics and bacterial growth medium to promote or inhibit growth. Growth profiles of both sensitive and resistant S. Aureus strains were measured on electrodes modified with gels containing different antibiotic concentrations to perform antibiotic susceptibility testing. The electrodes performed as expected, displaying growth where no antibiotic was present and no growth for sensitive $S$. aureus, and displaying growth in the case of low antibiotic concentrations (around the MIC values) for the resistant MRSA strain in $<45$ minutes. Such a system could be of enormous benefit in a clinical setting, enabling rapid antibiotic susceptibility testing for a range of clinically-relevant pathogens such as Gram-negative bacteria and fungi, performed at the point of care, ultimately improving infection detection and improving antibiotic stewardship. 


\section{Acknowledgements}

We would like to thank Professor Iain S. Hunter for discussions regarding MIC determination. The authors would like to thank Dr Catherine Breslin for project management and support.

\section{Funding}

DC would like to acknowledge MRC Confidence in Concept Scheme/R180246-103 for financial support and the National Endowment for Science Technology and Arts (NESTA) for financial support and award of a Longitude Prize Discovery Award to initially support this work in August 2017.

\section{References}

Barry, A. L., Reller, L. B., Miller, G. H., Washington, J. A., Schienknect, F. D., Peterson, L. R., Hare, R. S., Knapp, C., 1992. J. Clin. Microbiol. 30, 585-589.

Batchelor-McAuley, C., Katelhon, E., Barnes, E. O., Compton, R. G., Laborda, E., Molina, A., 2015. ChemistryOpen. 4, 224-260.

Brosel-Oliu, S., Mergel, O., Uria, N., Abramova, N., van Rijn, P., Bratov, A., 2019. Lab. Chip. DOI: $10.1039 / \mathrm{c} 81 \mathrm{c} 01220 \mathrm{~b}$.

Centers for Disease Control and Prevention, Laboratory Detection of: Oxacillin/MethicillinResistant Staphylococcus Aureus, At https://www.cdc.gov/hai/settings/lab/lab_mrsa.html accessed 07/05/19.

Chauhan, N., Chawla. S., Pundir, C. S., Jain, U., 2017. Biosensors and Bioelectronics. 89, 377383.

Chen, S., Duan, J., Tang, Y., Zhang Qiao, S., 2013. Chemistry. 19, 7118-7124.

Clarridge, J. E., 2004. Diseases. Clin. Microbiol. Rev. 17, 840-862.

Coll, F., Harrison, E. M., Toleman, M. S., Reuter, S., Raven, K. E., Blane, B., Palmer, B., 
Kappeler, A. R. M., Brown, N. M., Torok, M. E., Parkhill, J., Peacock, S. J., 2017. Sci. Transl. Med. 9, 1-19.

Davies, J., Davies, D., 2010. Microbio and Mol. Bio. Rev. 74, 417-433.

Department of Health and Social Care. The UK's Vision for AMR by 2040 and Five-Year National Action Plan, 2019.

https://ec.europa.eu/health/amr/sites/amr/files/ev_20190312_co05c_en.pdf

Du, C. X., Han, L., Dong, S. L., Li, L. H., Wei, Y., 2016. IOP Conf. Seri.: Mater. Sci. Eng. 137, 012060.

Gilligan, P. H., 2013. The Prokaryotes, Human Microbiology, $3^{\text {rd }}$ ed. Springer Reference, Heidelberg, New York, Dordrecht, London.

Han, L., Lu, X., Wang, M., Gan, D., Deng, W., Wang, K., Fang, L., Liu, K., Chan, C. W., Tang, Y., Weng, L. T., Yuan, H., 2017. Small. 13.

Harkins, C. P., Pichon, B., Doumith, M., Parkhill, J., Westh, H., Tomasz, A., de Lencastre, H., Bentley, S. D., Kearns, A. M., Holden, M. T. G., 2017. Genome. Biol. 18, 130.

Hayat, A., Marty, J. L., 2014. Sensors. 14, 10432-10453.

Henihan, G., Schulze, H., Corrigan, D. K., Giraud, G., Terry, J. G., Hardie, A., Campbell, C. J., Walton, A. J., Crain, J., Pethig, R., Templeton, K. E., Mount, A. R., Bachmann, T. T., 2016.

Biosensors and Bioelectronics. 81, 487-494.

Jiang, D., Ge, P., Wang, L., Jiang, H., Yang, M., Yuan, L., Ge, Q., Fang, W., Ju, X., 2019.

Biosensors and Bioelectronics. 130, 299-306.

Kuralay, F., Campuzano, S., Haake, D. A., Wang, J., 2015. Talanta. 85, 1330-1337.

McEwen, S. A., Collignon, P. J., 2018. Microbiol Spectr. 6. 
Michalek, J., Hobzova, R., Pradny, M., Duskova, M., 2010. Biomedical Applications of Hydrogels Handbook., 303-316.

National Institute for Health and Care Excellence. NICEimpact antimicrobial resistance. At https://www.nice.org.uk/Media/Default/About/what-we-do/Into-practice/measuringuptake/NICEimpact-antimicrobial-resistance.pdf (2018).

Ndiaye, A. L., Delile, S., Brunet, J., Varenne, C., Pauly, A., 2016. Biosensors. (Basel). 6, 46.

Nie, Z., Nijhuis, C. A., Gong, J., Chen, X., Kumachev, A., Martinez, A. W., Narovlyansky, M., Whitesides, G. M., 2010. Lab Chip. 10, 477-483.

O'Neill, J. Antimicrobial Resistance: Tackling a Crisis for the Health and Wealth of Nations, The Review on Antimicrobial Resistance chaired by Jim O'Neill. 2014. https://amrreview.org/sites/default/files/AMR\%20Review\%20Paper\%20\%20Tackling $\% 20 \mathrm{a} \% 20$ crisis $\% 20$ for $\% 20$ the $\% 20$ health $\% 20$ and $\% 20$ wealth $\% 20$ of $\% 20$ nations $1 . p$ df).

Russell, C., Ward, A. C., Vezza, V., Hoskisson, P., Alcorn, D., Steenson, D. P., Corrigan, D. K., 2019. Biosensors and Bioelectronics. 126, 806-814.

Sabath, L. D., Garner, C., Wilcox, C., Finland, M., 1976. Antimicrobial Agents and Chemotherapy. 9, 962-969.

Science Direct, Agarose - An Overview, At

https://www.sciencedirect.com/topics/chemistry/agarose accessed 07/05/19.

Settu, K., Liu, J. T., Chen, C. J., Tsai, J. Z., Chang, S. J., 2013. Conf. Proc. IEEE Eng. Med. Biol. Soc. 2013, 1712-1715. 
Song, Y., Xu, M., Gong, C., Shen, Y., Wang, L., Xie, Y., Wang, L., 2018. Sensors and Actuators B; Chemical. 257, 792-799.

Tan, C., Nasir, M. Z. M., Ambrosi, A., Pumera, M., 2017. Anal. Chem. 89, 8995-9001.

Tavakoli, J., Tang, Y., 2017. Mater. Sci. Eng. C. Mater. Biol. Appl. 77, 318-325.

Taylor, T. A., Unakal, C. G., 2019. StatPearls, Treasure Island Publishing.

Thermo Fisher Scientific, Agarose Gel Electrophoresis, At

https://www.thermofisher.com/uk/en/home/life-science/dna-rna-purification-analysis/nucleic-

acid-gel-electrophoresis/dna-electrophoresis/agarose-gel-electrophoreis.html accessed 07/05/19.

Todd, A., Worsley, A., Anderson, R. J., Groundwater, P. W., 2009. The Pharmaceutical Journal. $283,359-360$.

Tong, S. Y. C., David, J. S., Eichenberger, E., Holland, T. L., Fowler Jr, V. G., 2015. Clin. Microbiol. Rev. 28, 603-661.

Food and Agriculture Organization of the United Nations Report. Antimicrobial Resistance Policy Review and Development Framework. At http://www.fao.org/3/CA1486EN/ca1486en.pdf (2018).

Wang, W., Huang, H. Y., Chen, S. C., Ho, K. C., Lin, C. Y., Chou, T. C., Hu, C. H., Wang, W. F.,

Wu, C. F., Luo, C. H., 2011. Sensors. 11, 8593-8610.

Ward, A. C., Connolly, P., Tucker, N. P., 2013. PLOS One. 9, 91732. 
Ward, A. C., Hannah, A. J., Kendrick, S. L., Tucker, N. P., MacGregor, G., Connolly, P., 2018. Biosensors and Bioelectronics. 110, 65-70.

World Health Organization Global Action Plan on Antimicrobial Resistance. At https://apps.who.int/iris/bitstream/handle/10665/193736/9789241509763 eng.pdf?sequence=1 (2015).

\section{Author Contributions}

Stuart Hannah: Formal analysis, Investigation, Methodology, Project administration, Supervision, Validation, Visualisation, Writing - original draft, Writing - review and editing.

Emily Addington: Methodology, Formal analysis, Investigation, Methodology, Validation, Writing - Review and Editing. David Alcorn: Supervision, Funding Acquisition, Writing Review and Editing. Wenmiao Shu: Supervision, Funding Acquisition, Resources, Writing -

Review and Editing. Paul A. Hoskisson: Supervision, Funding Acquisition, Resources, Writing - Review and Editing. Damion Corrigan: Conceptualization, Formal analysis, Funding acquisition, Investigation, Methodology, Project administration, Resources, Supervision, Validation, Visualization, Writing - Review and Editing.

\section{Additional Information}

Supplementary information - Supplementary information is available at:

Competing interests - The authors declare no competing interests. 


\begin{tabular}{|c|c|c|c|c|c|c|c|c|c|c|}
\hline $\begin{array}{c}\text { Experiment: } \\
\text { Agarose } \\
\text { Concentration } \\
(\%)\end{array}$ & $\frac{R_{\underline{S O L N}}}{\underline{(\Omega)}}$ & 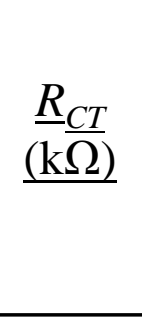 & $\underline{\underline{C_{D L}}} \underline{\underline{(\mu \mathrm{F})}}$ & $\chi^{2}$ & $\frac{\underline{Z \text { at }}}{\frac{100}{\mathrm{kHz}}}$ & $\frac{\text { Phase }}{\underline{\left({ }^{\circ}\right)}}$ & $\frac{\operatorname{DPVI}}{\underline{(\mu \mathrm{A})}} \underline{ }$ & $\frac{\mathrm{CV} \mathrm{i}}{\underline{(\mu \mathrm{A})}}$ & $\frac{\mathrm{CV} \Delta E_{p}}{\underline{(\mathrm{mV})}}$ & $\underline{\left.\frac{D}{\left(\mathrm{~cm}^{2}\right.} \mathrm{s}^{-1}\right)}$ \\
\hline $1 \%$ & 299.80 & 24.25 & 1.84 & 0.0007 & $\begin{array}{c}415.4 \\
0\end{array}$ & 22.42 & 0.46 & 0.69 & 110.40 & $4.15 \times 10^{-9}$ \\
\hline $1.5 \%$ & 731.40 & 94.03 & 0.57 & 0.0101 & $\begin{array}{c}658.5 \\
2\end{array}$ & 6.58 & 0.19 & 0.82 & 281.95 & $2.23 \times 10^{-6}$ \\
\hline $3.0 \%$ & 249.60 & 338.8 & 0.24 & 0.0011 & $\begin{array}{c}246.6 \\
5\end{array}$ & 6.18 & 0.02 & 0.41 & 498.52 & $7.64 \times 10^{-10}$ \\
\hline $\begin{array}{l}\text { Experiment: } \\
\text { Gel Deposition } \\
\text { Method }\end{array}$ & $\underline{R}_{\underline{S O L N}}^{\underline{(\Omega)}}$ & $\frac{\underline{R}_{C T}}{\underline{(\mathrm{k} \Omega})}$ & $\frac{\underline{C}_{D L}}{(\mu \mathrm{F})}$ & $\chi^{2}$ & $\frac{\underline{Z \text { at }}}{\frac{100}{\mathrm{kHz}}}$ & $\frac{\text { Phase }}{\underline{\left({ }^{\circ}\right)}}$ & $\frac{\mathrm{DPV} I}{(\mu \mathrm{A})}$ & $\frac{\mathrm{CV} \mathrm{i}}{p k}$ & $\frac{\mathrm{CV} \Delta E_{p}}{(\mathrm{mV})}$ & $\begin{array}{c}\frac{D}{\left(\mathrm{~cm}^{2} \mathrm{~s}^{-1}\right)} \\
\end{array}$ \\
\hline Dip Coat & 226.50 & I & 2.04 & 0.0024 & $\begin{array}{c}217.4 \\
2\end{array}$ & 1.66 & 5.26 & I & I & I \\
\hline $\begin{array}{l}\text { Drop Cast } \\
\quad(10 \mu \mathrm{l})\end{array}$ & 191.40 & 0.08 & 3.38 & 0.0008 & $\begin{array}{c}185.6 \\
4\end{array}$ & 1.87 & 2.91 & 0.102 & 140.94 & I \\
\hline $\begin{array}{c}\text { Drop Cast } \\
(20 \mu \mathrm{l})\end{array}$ & 242.2 & 3.91 & 1.36 & 0.0009 & $\begin{array}{c}242.4 \\
1\end{array}$ & 4.37 & 1.42 & 1.26 & 85.55 & I \\
\hline
\end{tabular}

Table 1. Sumn

shown in Figure

shows CV, EIS a

measurement dat

from gel develop

experiments. Me

parameters inclu

$C_{D L}, Z(100 \mathrm{kHz})$

$I_{p k}, C V i_{p k}, C V \Delta t$ 
a (ii)

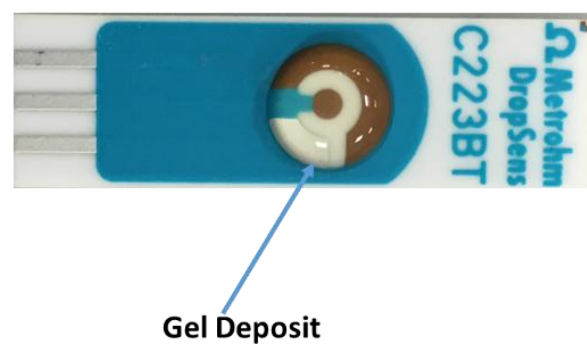

a (iii)

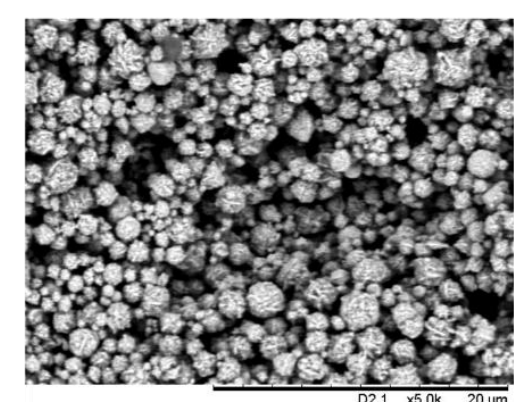

b

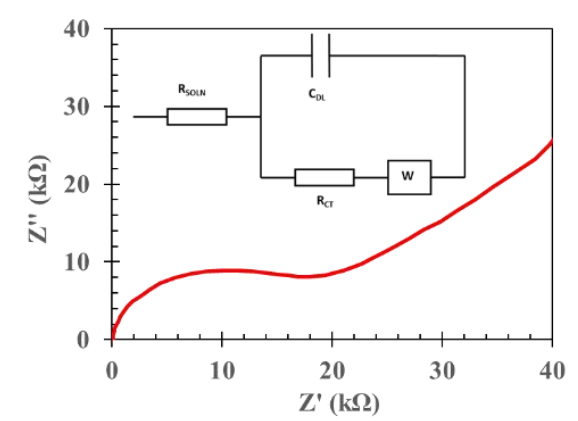

c (i)

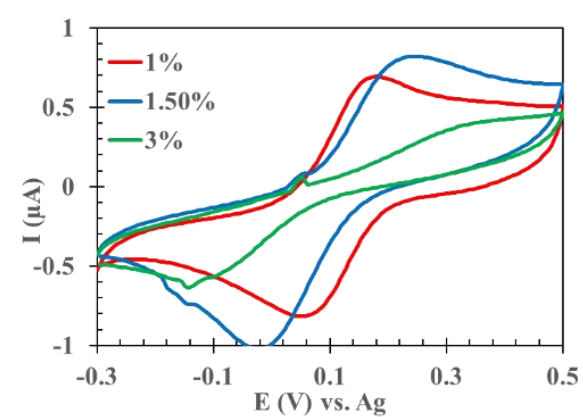

d (i)

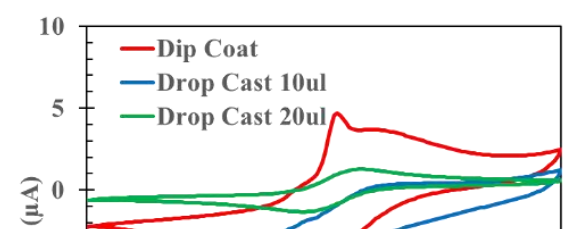

a (iv)

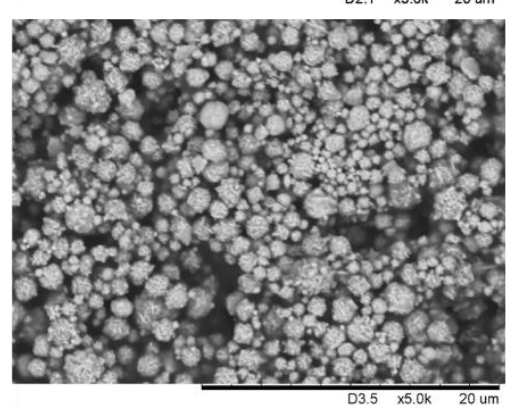

c (ii)

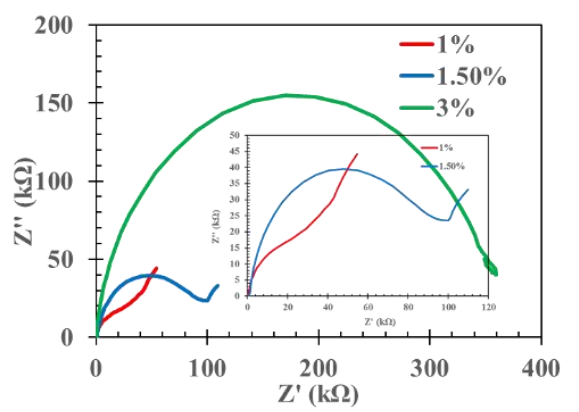

d (ii)

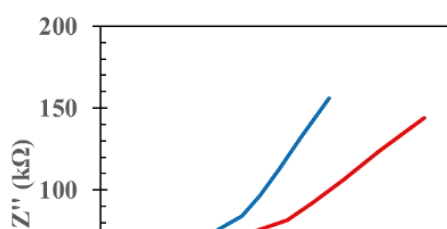

FF-C and some with antibiotic. Schematic depicts bacteria beir pipetted onto electrode, and electrochemical measurements being performed using a potentiostat. (a) (ii) Electrode surface modified with agarose gel deposit. (a) (iii) SEM image of bare Au DropSens electrode. (a) (iv) SEM image of Au DropSens electrode with $1 \%$ agarose gel deposit. (b) Typical electrochemical impedance spectroscopy (EIS) measurement o 1 mM FF-C on Au SPE. Inset shows Randles' equivalent circui model used to fit EIS data. (c) CV (i) and EIS (ii) measuremen on different concentrations of agarose gel containing F-F $+\mathrm{KC}$ Inset in (c) (ii) shows 1 and $1.5 \%$ gels only for clarity. (d) CV (i) and EIS (ii) measurements comparing gel deposition 
a (i)

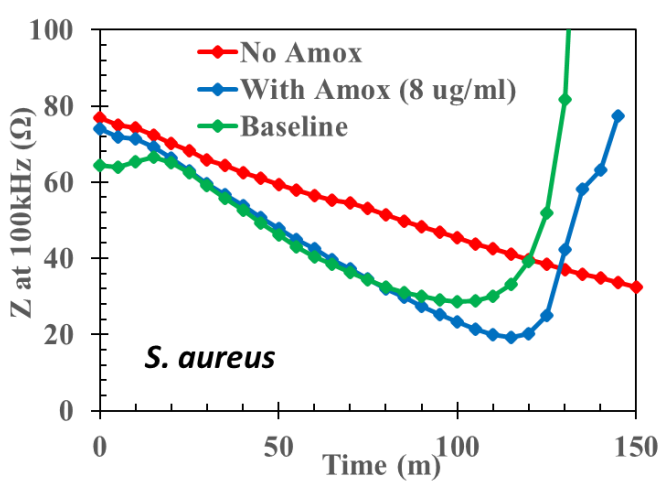

b (i)

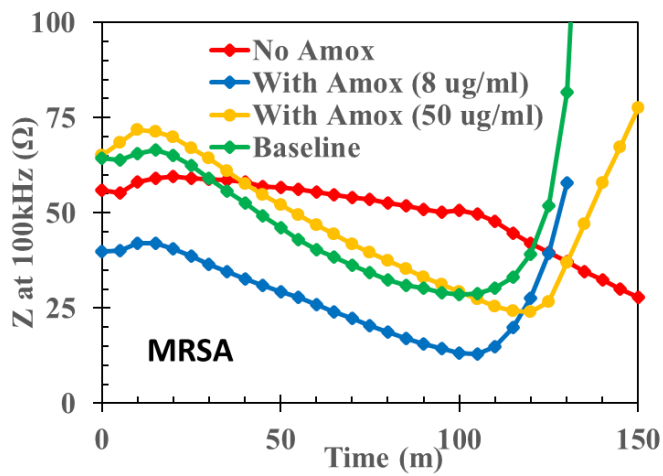

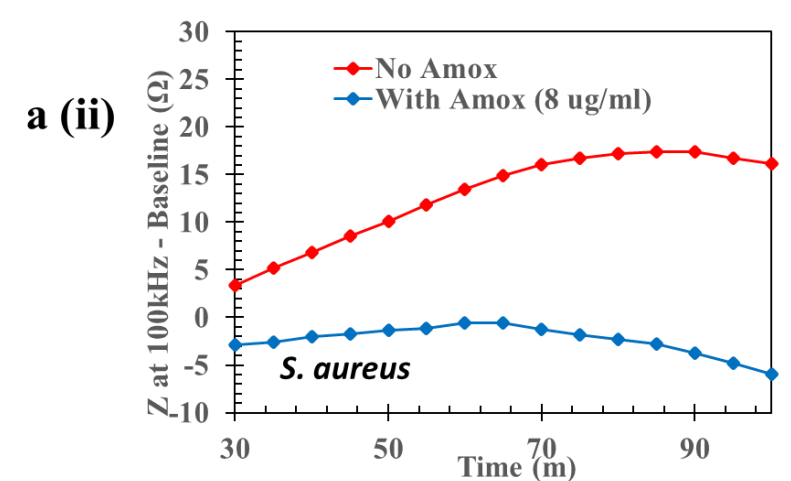

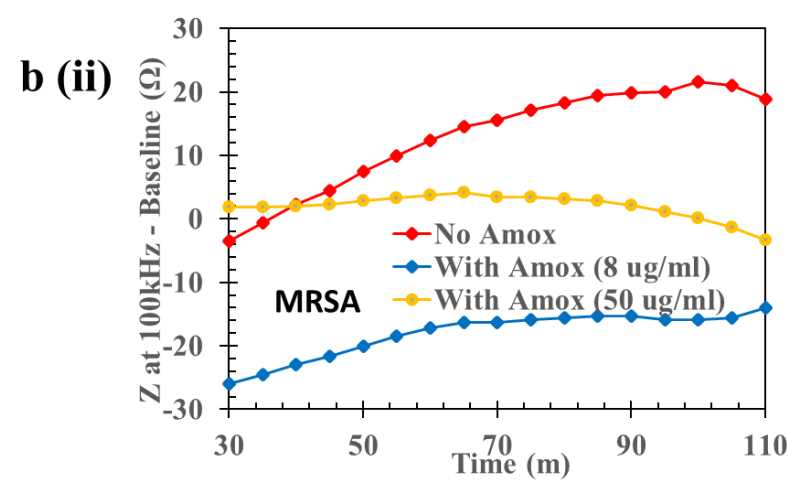

Figure 2. Bacterial growth curves of sensitive $S$. at and MRSA (b) strains on gels infused with and with amoxicillin $(8 \mu \mathrm{g} / \mathrm{ml}$ (and $50 \mu \mathrm{g} / \mathrm{ml}$ for MRSA)) anc (no bacteria). (i) $\mathrm{Z}$ at $100 \mathrm{kHz}$ and (ii) $\mathrm{Z}$ at $100 \mathrm{kHz}$ curve. 

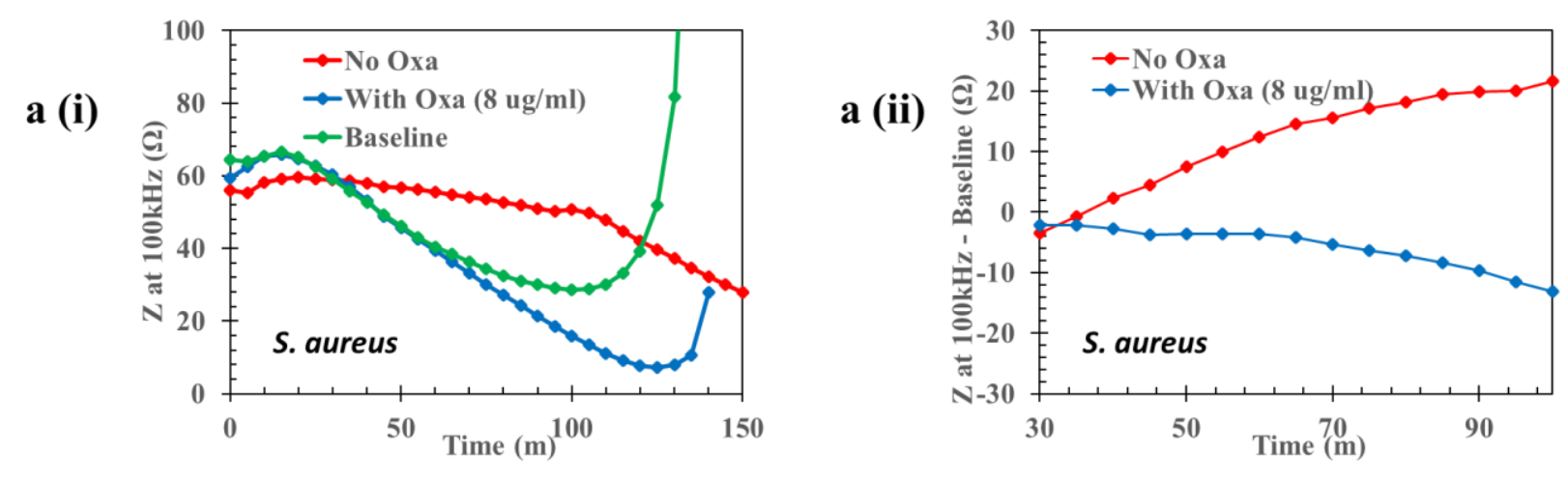

Figure 3. Bacteria growth curves of sensitive $S$. aureu and MRSA (b) strains on gels infused with and without (Oxa) $(8 \mu \mathrm{g} / \mathrm{ml})$ and baseline (no bacteria). (i) $\mathrm{Z}$ at 100

(ii) $\mathrm{Z}$ at $100 \mathrm{kHz}$ - baseline curve.
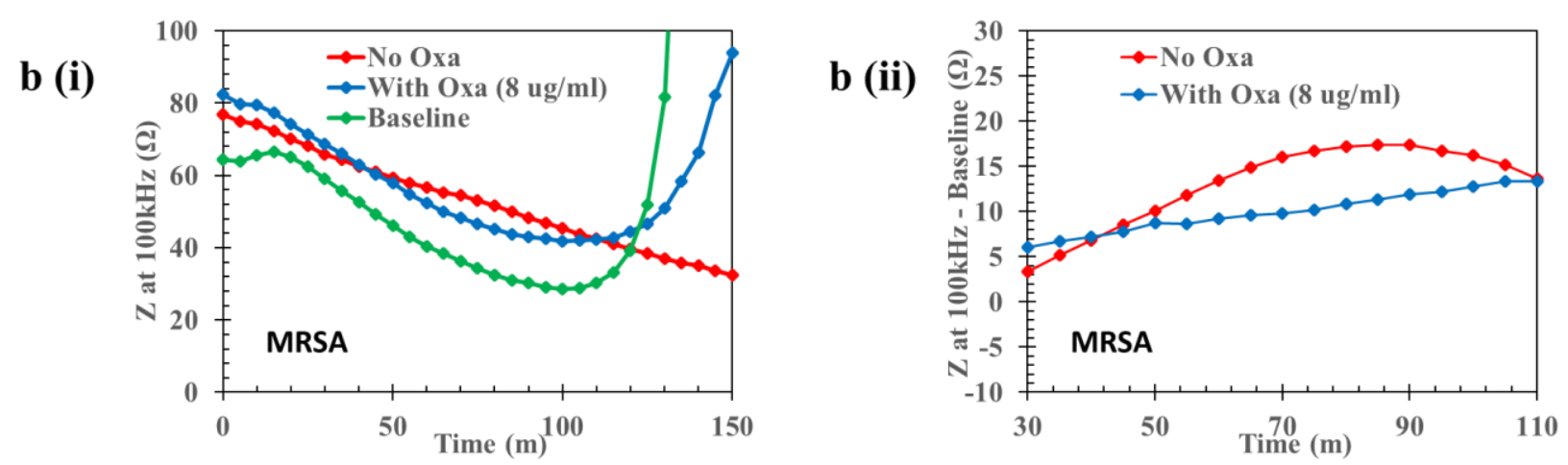


\section{Screen \\ Printed}

Electrode

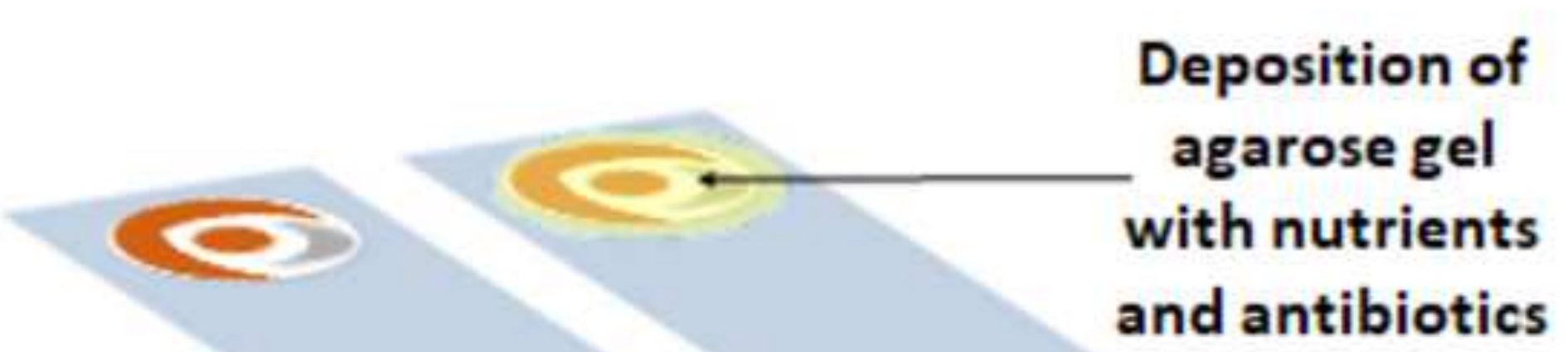

S.Aureus

Loading of bacteria onto antibiotic-gel modified electrodes S.Aureus vs MRSA

Electrochemical Signal Change-Growth Curves

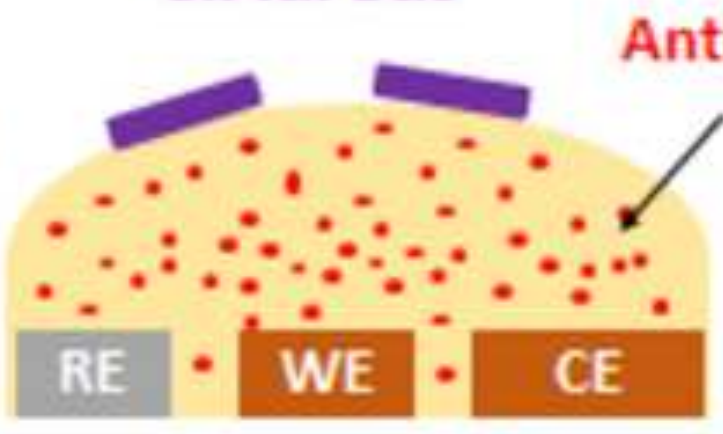

S.Aureus - no growth

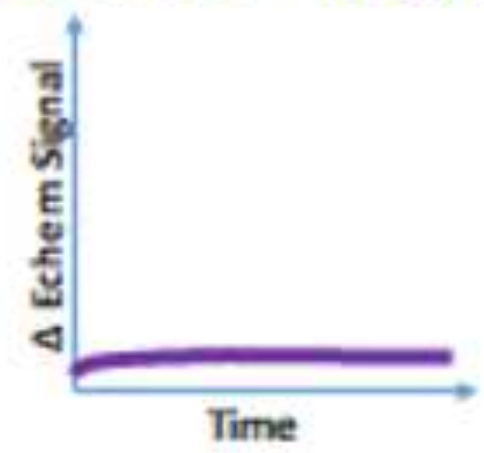

MRSA
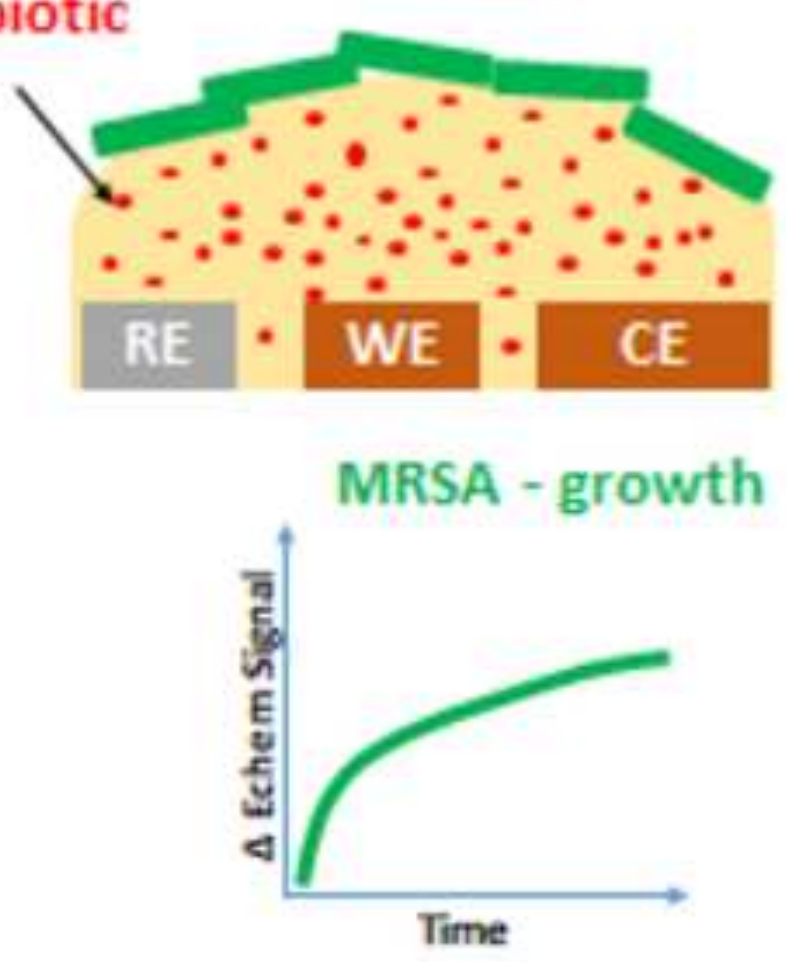
Supplementary Material

Click here to download Supplementary Material: Supporting Information - Final (After Peer Review).pdf

(After Peer Review).pdf

(1)

-

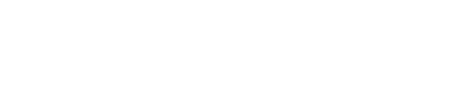

-

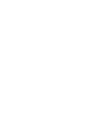

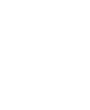

$-$

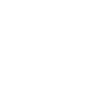

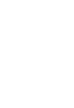

.


- A gold screen printed electrode modified with a hydrogel deposit was developed.

- The sensor was electrochemically characterised to monitor bacterial growth.

- Growth profiles were developed for susceptible and drug resistant S. aureus.

- Rapid growth profiles established; antibiotic susceptibility results $<45$ minutes. 
Stuart Hannah: Formal analysis, Investigation, Methodology, Project administration, Supervision, Validation, Visualisation, Writing - original draft, Writing - review and editing. Emily Addington: Methodology, Formal analysis, Investigation, Methodology, Validation, Writing - Review and Editing. David Alcorn: Supervision, Funding Acquisition, Writing - Review and Editing. Wenmiao Shu: Supervision, Funding Acquisition, Resources, Writing - Review and Editing. Paul A. Hoskisson: Supervision, Funding Acquisition, Resources, Writing - Review and Editing. Damion Corrigan: Conceptualization, Formal analysis, Funding acquisition, Investigation, Methodology, Project administration, Resources, Supervision, Validation, Visualization, Writing - Review and Editing. 


\title{
Declaration of interests
}

$\bigotimes$ The authors declare that they have no known competing financial interests or personal relationships that could have appeared to influence the work reported in this paper.

$\square$ The authors declare the following financial interests/personal relationships which may be considered as potential competing interests:

\author{
Stuart Hannah \\ Emily Addington \\ David Alcorn \\ Wenmiao Shu \\ Paul A. Hoskisson \\ Damion K. Corrigan
}

